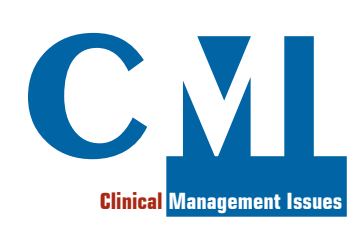

Clinical Management

\title{
Novel Therapies in HBV Infection
}

\begin{abstract}
Current treatments for chronic hepatitis B are able to provide a sustained suppression of the viral replication (i.e., persistent undetectability of $H B V D N A$ ). This leads to improvement of liver fibrosis and reduction of clinical complications. However, hepatitis B surface antigen (HBs $A g$ ) persists in most patients, probably justifying a still increased risk of hepatocellular carcinoma. Indeed, obtaining a complete and sterilizing cure with elimination of the covalently closed circular DNA (cccDNA) or silencing its activity is still a holy grail. Nerw molecules are under evaluation to suppress viral replication acting on multiple phases of the HBV cycle or improve specific immune response against $H B V$. Molecules acting on $H B V$ cycle have already showed encouraging results, such as entry inhibitors, small interfering $R N A s$ (siRNAs), capsid assembly modulators (CAMs), nucleic acid polymers (NAPs). Also, promising results have been observed with immune-modulators, therapeutic vaccines, and other immune-based approaches. Among these, toll-like (TLR) or anti-programmed receptor agonists antibody 1 of the cell death protein (PD1) (e.g., nivolumab) are most promising. This paper describes newer drugs appearing on the horizon, including antiviral drugs targeting different steps of the HBV life cycle and therapeutic approaches based on immune-modulation.
\end{abstract}

Keywords: Antiviral Therapy; Hepatitis B Surface Antigen;Nucleos(t)ide Analogs; PEGylated Interferon; Covalently Closed Circular DNA; Immune-Modulatory Therapy

CMI 2019; 13(1): 53-60

http://dx.doi.org/10.7175/cmi.v13i1.1434

\section{INTRODUCTION}

In contrast to the three main pandemic infectious diseases such as tuberculosis, malaria, and HIV/AIDS, mortality for viral hepatitis rose between 2000 and 2015 , being mortality attributable to chronic HBV infection the most prevalent [1]. HBV infection is a major cause of liver cirrhosis and hepatocellular carcinoma (HCC). Although $\mathrm{HBV}$ infection can effectively be prevented by vaccination, there is no eradicating cure for those who acquired the infection.

The main clinical goals of therapy for chronic hepatitis $\mathrm{B}(\mathrm{CHB})$ are:

1. improvement of survival by preventing cirrhosis and end-stage liver diseases;
2. improvement of patient quality of life (QOL).

In clinical practice, the most validated surrogate outcomes are: HBV DNA suppression, hepatitis $\mathrm{B}$ e antigen $(\mathrm{HBeAg})$ clearance and relative sero-conversion, hepatitis B surface antigen ( $\mathrm{HBsAg}$ ) loss and $\mathrm{HBsAb}$ sero-conversion, aspartate transaminase (AST) and alanine transaminase (ALT) normalizations, and histological improvement (i.e., fibrosis reduction) [2,3]. Based on these data, three definitions of $\mathrm{HBV}$ cure are proposed, such as complete, functional and partial.

Complete cure is represented by undetectable $\mathrm{HBsAg}$ in serum and eradication of HBV DNA, including intrahepatic cova-
${ }^{1}$ Unità Operativa Malattie Infettive, Grande Ospedale Metropolitano "BianchiMelacrino-Morelli”, Reggio Calabria, Italy 2 Unità Operativa Malattie Infettive e Tropicali, Dipartimento di Scienze Mediche e Chirurgiche, Università degli Studi "Magna Graecia" di Catanzaro, Catanzaro, Italy

Corresponding author Giuseppe Foti giuseppe.foti@ospedalerc.it

Received: 19 April 2019 Accepted: 2 December 2019 Published: 12 December 2019 
lently closed circular DNA (cccDNA) and integrated HBV DNA.

Functional cure is defined by sustained $\mathrm{HBsAg}$ loss (with or without HBsAb sero-conversion), undetectable serum $\mathrm{HBV}$ DNA, persistence of cccDNA in a transcriptional inactive status, and the absence of spontaneous relapse after cessation of treatment.

Lastly, partial cure is characterized by detectable $\mathrm{HBsAg}$, but persistently undetectable HBV DNA in serum after a finite course of treatment $[4,5]$.

Current treatments for hepatitis $\mathrm{B}$ are pegylated-interferons (Peg-IFNs) and nucleos $(\mathrm{t})$ side analogs (NAs).

Peg-IFN can determine a sustained virological response (SVR), but only in selected patients and in a small percentage of cases. Moreover, Peg-IFN can improve immune control of HBV infection, with satisfactory rates of $\mathrm{HBeAg}$ sero-conversion but only in few cases hepatitis B surface antigen (HB$\mathrm{sAg}$ ) is cleared, albeit in such cases HBV DNA suppression is achieved [6,7].

Use of nucleos $(t)$ ide analogs achieves HBV DNA suppression. However, their administration, in most cases, must be continued long-life. Consistent data demonstrated that long-term control of $\mathrm{HBV}$ replication by the most potent drugs (entecavir-ETV and tenofovir-TDF) improves liver histology, with a decreased risk of progression to cirrhosis, end-stage liver disease, HCC and improved long-term survival [8]. Whilst these drugs obtain long-term suppression of HBV DNA in plasma, they are not able to obtain elimination or stable silencing of the cccDNA expression.

This paper describes newer drugs appearing on the horizon, including antiviral drugs targeting different steps of the HBV life cycle and therapeutic approaches based on immune-modulation. Probably, the most efficient use of these drugs is in combination.

\section{ANTIVIRAL DRUGS TARGETING DIFFERENT STEPS OF HBV LIFE CYCLE}

\section{Entry inhibitors}

Myrcludex B (MyrB), also known as bulevirtide, is a lipopeptide derived by a portion of the preS1 domain of HBV L-surface protein. It blocks the sodium taurocholate cotransporting polypeptide (NTCP), which is an essential step for HBV entry [9-11]. This drug has initially been used for treatment of HBV/HDV co-infected patients, aiming at better control of HDV replication $[12,13]$. With this objective in mind, a multicenter, open-label, phase 2 clinical trial was conducted. In this trial, MyrB significantly reduced HDV RNA levels in serum and induced ALT normalization $[14,15]$. Although adverse events were reported mostly as mild or moderate, elevated plasma bile acid levels were found as a typical effect of this drug. This effect was due to blocking of NTCP mediated reuptake of circulating bile acids from the portal blood into the liver [16]. Indeed, a prospective trial on 12 healthy volunteers treated with TDF and MyrB showed substantially increased plasma bile acids but this was not associated with any clinical symptoms typically attributed to cholestasis such as pruritus or steatorrhea [17]. Owing to its mechanism of action, it is unlikely that $\mathrm{MyrB}$ will provide sustained $\mathrm{HBsAg}$ decrease, at least when used alone. Moreover, one can hardly conclude that this drug would provide significant benefits in terms of cccDNA reduction.

\section{Small interfering RNAs (siRNAs)}

Small interfering RNAs (siRNAs) are small synthetic RNA molecules able to recognize complementary sequences of viral messenger RNA (mRNA) and pregenomic RNA (pgRNA). This induces mRNA degradation mediated by endonuclease enzymes.

ARO-HBV (JNJ3989) was evaluated in a phase II, multi-dose escalating study in patients with chronic HBV infection. This study showed that three monthly doses of ARO-HBV, administered subcutaneously, resulted in a median reduction of circulating $\mathrm{HBs} \mathrm{Ag}$ of approximately $2.0 \log _{10}$ at the third month and all treated patients showed a reduction greater than $1.0 \log _{10}$. The drug was well tolerated, with generally mild and self-limiting injection site adverse events in approximately $10 \%$ of cases [18]. ARB-1467 was tested in another phase II study, showing > $1 \log _{10}$ reduction of $\mathrm{HB}-$ sAg in 7/11 study patients. HBsAg reductions were dose-dependent, since they were greater with $0.4 \mathrm{mg} / \mathrm{kg}$ doses than with $0.2 \mathrm{mg} / \mathrm{kg}$ [19]. Also in this case, given the post-transcriptional site of action, it is unlikely that cccDNA reduction is achievable with the use of these drugs as mono-therapy. Moreover, the number of patients studied was extremely limited, thus further clinical 
investigations are needed with both AROHBV and ARB-1467.

\section{Capsid assembly modulators (CAMs)}

Capsid assembly modulators (CAMs) are small molecules, heterogenic for chemical structure (phenylpropenamides and sulfamoylbenzamides), which inhibit $\mathrm{HBV}$ pgRNA encapsidation, an essential step for the subsequent viral DNA synthesis.

In recent years, several CAMs have been studied such as ABI-H0731, JNJ-6379, and NVR 3-778. All three compounds led to a significant decline in HBV DNA, but with smaller reductions of HBV RNA and quantitative $\mathrm{HBsAg}$ (qHBsAg). Importantly, NVR 3-778 was used in association with Peg-IFN, leading to greater reduction of HBV DNA [20-22].

\section{Nucleic acid polymers (NAPs)}

Nucleic acid polymers (NAPs) are broad spectrum antiviral agents whose mechanism of action is not well defined. Their antiviral activity in $\mathrm{HBV}$ infection may be due to blocking of $\mathrm{HBsAg}$ release from hepatocytes. After a lead-in therapy with TDF, 40 patients were randomized into an experimental group (48 weeks of TDF + PegIFN and REP 2139-Mg or REP 2165-Mg) or an adaptive control group (24 weeks of TDF + Peg-IFN followed by 48 weeks of experimental therapy including REP 2139$\mathrm{Mg}$ or REP 2165-Mg) [23]. The majority of participants (more than 80\%) achieved either HBV DNA < $2000 \mathrm{IU} / \mathrm{ml}$ with normal transaminase levels or functional cure with undetectable HBsAg and HBV DNA. Although a substantial proportion of patients showed transaminase flares, they were correlated with better treatment response, probably reflecting immune-clearance of infected hepatocytes.

There results are encouraging, however since the drug may promote accumulation of HBsAg into hepatocytes, it is unclear whether such accumulation is safe. Indeed, $\mathrm{HBsAg}$ could display oncogenic potential which may impact on risk of HCC over the long term [23-25].

\section{Candidate therapeutic strategies to target covalently closed circular DNA (cccDNA)}

Inhibition of cccDNA formation could be used in theory to eradicate $\mathrm{HBV}$ infection.
However, many of the key steps in cccDNA formation require host nuclear molecules like enzymes and nuclear histones [26,27], but targeting host cell molecules might have several adverse effects. More studies should be conducted to better understand if a virushost protein interaction could be targeted without significant adverse events.

Once formed, cccDNA can potentially be targeted by nucleases or transcription activator-like effector nucleases. The development of CRISPR-Cas9 technology (clustered regularly interspaced short palindromic repeats) may be useful to edit $\mathrm{HBV}$ cccDNA. Preclinical experiments showed that CRISPR-CAS9-based strategies determined mutations and deletions which functionally inactivated cccDNA and were able to clear more than $90 \%$ of HBV DNA $[28,29]$.

Control of the transcriptional activity could be obtained by silencing of cccDNA through the induction of the host cell epigenetic machinery using general epigenetic modifiers. However this effect may be associated with adverse events on cell homeostasis.

$\mathrm{HBV} \mathrm{X}$ protein $(\mathrm{HBx})$ is a therapeutic target of potential interest. It is implicated in cccDNA stability and expression, interacting with damaged DNA binding protein (DDB1), redirecting the ubiquitin ligase activity of the CUL4-DDB1 E3 complexes against SMC 5/6 complexes, therefore suppressing $\mathrm{HBV}$ transcription. $\mathrm{HBx}-\mathrm{DDB} 1$ interaction may be used to block cccDNA transcription and several molecules have been studied. Pevonedistat, a NEDD8-activating enzyme inhibitor, could restore SMC 5/6 levels and suppress viral transcription and protein production in cultured hepatocytes [30,31]. Nitazoxanide, a thiazolide agent already approved by the US Food and Drug Administration for protozoan enteritis, showed efficient inhibition of the $\mathrm{HBx}-$ DDB1 interaction, restoring SMC5 levels and suppressing viral transcription and protein production in cultured hepatocytes [30]. A pilot clinical trial in 9 patients showed undetectable HBV DNA (<38 IU/ml) in 8/9 patients after 4-20 weeks of treatment [32].

In conclusion, while several mechanisms have been proposed to eradicate or silence cccDNA expression (synthesis control, control of transcriptional activity and genome clearance), such strategies are limited by the stability of cccDNA in host cells, and for the difficulty in targeting host molecules without producing significant adverse events. 


\section{IMMUNE-MODULATORS}

In chronic $\mathrm{HBV}$ infection, prolonged exposure to viral antigens is associated with functional impairment of the immune response against this virus, both systemically and locally within the liver [33]. Therefore, development of drugs able to restore immune response against HBV may be very useful. However, since the liver milieu may be profoundly de-regulated in patients with $\mathrm{CHB}$, such immune-therapy strategies may not work. So, despite quite favorable data, it remains to be determined in which conditions immune-therapy should be pursued. It is possible that antiviral therapy should precede immune-therapy to reduce $\mathrm{HBV}$ DNA and hence liver inflammation which may favor response to immune-therapy. Also, treating patients at early stages of the infection when inflammation and fibrosis are still absent or mild (e.g., noninflammatory stages or immune-tolerance phase of the infection) may be beneficial.

Compounds for immune-therapy are already under study, acting either on nonspecific innate immunity or specific adaptive immunity against HBV. Among the first group of compounds acting on nonspecific innate immunity, immune stimulators, such as inarigivir, and toll-like receptor agonists (TLR), such as GS-9620 (TLR7) and GLS9688 (TLR8) have been studied. Among the second group of compounds, which may boost specific response, therapeutic vaccines (e.g., GS-4774) and other therapeutic approaches such as anti-programmed cell death protein 1 (anti-PD-1) antibodies (e.g., nivolumab) were object of important studies. Probably, associations of these two types of drugs with or without antiviral therapy is the next step of HBV treatment if eradication of this infection is pursued.

\section{Drugs acting on non-specific immune response}

Inarigivir is a novel oral selective immunemodulator with associated antiviral activity. It acts as a retinoic acid-inducible gene-I (RIG-I) agonist, inducing type I and type III interferon (IFN) intracellular production in order to boost immune-response. Also, a direct antiviral activity was observed through interference with $\mathrm{HBV}$ polymerase transcription [34]. A dose-related reduction of HBV DNA and RNA levels (0.58-1.54 $\log$ decrease) was observed in human trials, particularly in $\mathrm{HBeAg}$-negative patients.
Also qHBsAg levels were reduced, 16/49 patients obtaining reduction in $\mathrm{qHBs} A \mathrm{~g}$ levels at week 24 by more than $0.5 \log$ [35]. Activation of systemic innate or adaptive immune responses was also observed, especially with higher drug dosages [36].

The toll-like receptor (TLR) agonists promote up-regulation of type 1 interferon and other cytokines, activating natural killer cells and enhancing innate immunity against HBV. GS-9620 is a TLR7 agonist. Strong antiviral activity was observed in HBV-infected chimpanzees and woodchucks, but this effect was transient. Clinical trials on GS-9620 were conducted showing no side effects on humans but little antiviral activity. However, it is possible that this evidence is related to suboptimal dosages used in $\mathrm{pa}^{-}$ tients: $4 \mathrm{mg}$ vs. $1 \mathrm{mg} / \mathrm{kg}$ in chimpanzees. Dose-related expression of interferon-stimulated gene 15 (ISG15) was also observed, but there was no significant decline of serum HBsAg levels [37-39]. GS-9688, a potent and selective, oral, small-molecule agonist of TLR- 8 was evaluated in a phase $1 \mathrm{~b}$ clinical trial in patients with chronic virally suppressed CHB. Favorable immunological responses were observed (increase in IL-12p40 and IL1RA) but no significant changes of HBV replication were obtained [40].

\section{Drugs acting on specific immune response}

GS 4774 is a therapeutic vaccine engineered to activate an $\mathrm{HBV}$-specific $\mathrm{T}$ cell immune response. It was experimented at three different dosages in six administrations for a total of 20 weeks in combination with oral antiviral therapy (OAV), compared with a control group of OAV only. Efficacy was measured by $\mathrm{HBs} \mathrm{Ag}$ decline from baseline to week 24 . There were no significant differences among groups in the mean HB$\mathrm{sAg}$ decline from baseline to week 24 or 48, although five $\mathrm{HBeAg}$-positive patients receiving GS-4774 experienced HBeAg loss versus none in the control group. The treatment was generally safe and well tolerated, with no serious adverse events or discontinuations reported [41].

Check point inhibitors (anti-PD-1) are able to determine a functional restoration of peripheral and intrahepatic exhausted HBV specific T cells. Gane et al. conducted a phase 1 clinical study in 8 patients treated with nivolumab with or without GS-4774 and observed that a single dose of nivolumab up to $0.3 \mathrm{mg} / \mathrm{kg}$ was well tolerated. A signifi- 
cant decline in $\mathrm{qHBsAg}$ in patients receiving nivolumab was also observed, with no added benefit using also GS-4774 [42]. Recently, another trial showed $\mathrm{qHBsAg}$ reduction and in one patient becoming undetectable at week 20 even if an hepatitis flare was observed accompanied by a significant increase in peripheral HBsAg-specific T cells [43].

\section{Human monoclonal antibodies and TCR/ CAR-T cells}

Promising results were obtained with monoclonal antibodies (mAbs) and TCR/ CAR-T cells. This approach is likely to be complementary to antiviral treatment and has been extensively reviewed by Cerino et al. [44]. Current evidence suggests that both $\mathrm{T}$ and $\mathrm{B}$ cell responses are necessary to obtain HBV eradication. Supporting this hypothesis is that HBV vaccines and $\mathrm{Ab}$ serum may protect exposed patients from new infections (e.g., newborns from infected mothers). However, this effect is only possible when vaccines and $\mathrm{Ab}$ serum are given early, while impact of such strategies in patients already infected and with a viral reservoir already established into hepatocytes is not supported by data. Importantly, current research aims at finding specific epitopes to be targeted more effectively. Interestingly, a linear epitope (aa 119-125 of $\mathrm{HBsAg}$ ) may promote $\mathrm{HBV}$ clearance and block new rounds of infection [45]. Also, in murine models, humAbs targeting the NTCP-binding site of preS1 reduced HBV viremia and $\mathrm{HBsAg}$ levels [46].

Adoptive transfer of lymphocytes expressing engineered T cell receptors (TCRs) is a promising immunotherapeutic option which specifically targets antigens from viral-in- fected cells and tumors. These TCR-T cells may recognize HBV epitopes presented on infected hepatocytes and even HCC cells with HBV-DNA integration in both experimental models and selected patients with $\mathrm{HBV}$-related HCC relapses. However, safety concerns on possible irreversible structural and functional liver damage by cytotoxic TCR-T cells have limited the clinical usage of this immunotherapeutic approach. Modified TCR-T cells to reduce cytotoxicity and life span of TCR-T cell could reduce this safety concern $[47,48]$.

\section{CONCLUSIONS}

Despite the numerous therapeutic approaches by using drugs targeting different steps of the HBV life cycle and immunemodulating strategies, to date there are no validated alternatives to current antivirals or Peg-interferon.

However, it is important to continue searching and experimenting new compounds. Also, the way forward appears to be combination of drugs acting on different mechanisms (e.g., immune-therapy approach plus antivirals). With this objective in mind, more strategic studies should be conducted.

Lastly, we need to know what patients are better suited to achieve a functional cure. Probably, patients at earlier stages of infections are the best candidates, implying that strategies to achieve earlier diagnoses should be pursued. Until these possibilities are realistic, we may strengthen more on prevention, assuring vaccination and safe behaviors (e.g., safe sex) to the largest number of individuals as possible.

Key points

- Mortality for complication or sequelae of viral hepatitis rose between 2000 and 2015, being $H B V$ infection largely prevalent.

- Interferon therapy, which is poorly tolerated due to frequent adverse events, is effective only in patients who are carefully pre-selected on the basis of predictive criteria (pre-therapy and stopping rules during therapy).

- The NAs, although well tolerated should be administered life-long in most cases.

- The current anti-HBV therapies achieve a functional cure only rarely and complete cure characterized by destruction of the cccDNA or stable silencing of its activity has not been demonstrated yet.

- Therefore, new drugs ensuring functional cure are needed and not far away.

- Probably, it is only through combination of different strategies (e.g., antivirals and immune-modulators) and by a careful selection of patients treated in the early stages of the $H B V$ infection that we will move towards a new era in HBV treatment. 


\section{Funding}

This article has been published without the support of sponsors.

Conflicts of interests

The author declare he has no competing financial interests concerning the topics of this article.

\section{REFERENCES}

1. WHO Global Health Estimates 2015. Deaths by cause, age, sex, by country and by region, 2000-2015. Geneva: World Health Organization, 2016

2. EASL. Clinical practice guidelines on the management of hepatitis B virus infection.J Hepatol 2017; 67: 370-98; https://doi.org/10.1016/j.jhep.2017.03.021

3. Terrault NA, Bzowej NH, Chang KM, et al. AASLD guidelines for treatment of chronic hepatitis B. Hepatology 2016; 63: 261-83; https://doi.org/10.1002/hep.28156

4. Lok AS, Zoulim F, Dusheiko G, et al. Hepatitis B cure: from discovery to regulatory approval. J Hepatol 2017; 67: 847-61; https://doi.org/10.1016/j.jhep.2017.05.008

5. Zoulim F. Inhibition of hepatitis B virus gene expression: a step towards functional cure. $J$ Hepatol 2018; 68: 386-8; https://doi.org/10.1016/j.jhep.2017.11.036

6. Marcellin P, Bonino F, Yurdaydin C, et al. Hepatitis B surface antigen levels: association with 5 year response to peginterferon alfa-2a in hepatitis B e-antigen-negative patients. Hepatol Int 2013; 7: 88-97; https://doi.org/10.1007/s12072-012-9343-x

7. Kao JH. HBeAg-positive chronic hepatitis B: why do I treat my patients with pegylated interferon? Liver International 2014; 35 (Supp. 1): 112-9; https://doi.org/10.1111/liv.12400

8. Marcellin P, Asselah T. Long-term therapy for chronic hepatitis B: hepatitis B virus DNA suppression leading to cirrhosis reversal. J Gastroenterol Hepatol 2013; 28: 912-23; https://doi. org/10.1111/jgh.12213

9. Ni Y, Lempp FA, Mehrle S, et al. Hepatitis B and D viruses exploit sodium taurocholate cotransporting polypeptide for species-specific entry into hepatocyte. Gastroenterology 2014; 146: 1070-83; https://doi.org/10.1053/j.gastro.2013.12.024

10. Schulze A, Gripon P, Urban S. Hepatitis B virus infection initiates with a large surface proteindependent binding to heparansulfateproteoglycans. Hepatology 2007; 46: 1759-68; https://doi. org/10.1002/hep.21896

11. Schieck A, Schulze A, Gähler C, et al. Hepatitis B virus hepatotropism is mediated by specific receptor recognition in the liver and not restricted to susceptible hosts. Hepatology 2013; 58: 43-53; https://doi.org/10.1002/hep.26211

12. Bogomolov $\mathrm{P}$, Alexandrov A, Voronkova N, et al. Treatment of chronic hepatitis $\mathrm{D}$ with the entry inhibitor myrcludex B: First results of a phase Ib/IIastudy. J Hepatol 2016; 65: 490-8; https://doi.org/10.1016/j.jhep.2016.04.016

13. Blank A, Markert C,Hohmann N, et al. First-in-human application of the first-in-class hepatitis B and hepatitis D virus entry inhibitor myrcludex B. J Hepatol 2016; 65; 483-9; https://doi. org/10.1016/j.jhep.2016.04.013

14. Wedemeyer H, Bogomolov P, Blank A, et al. Final results of a multicenter, open-label phase $2 \mathrm{~b}$ clinical trial to assess safety and efficacy of Myrcludex B in combination with Tenofovir in patients with chronic HBV/HDV co-infection.J Hepatology 2018; 68 (Suppl 1): s3; Abstr GS-005; https://doi.org/10.1016/S0168-8278(18)30224-1

15. Wedemeyer H, Schöneweis K, Bogomolov P, et al. Interim Results of a Multicentre, Open-Label Phase 2 Clinical Trial (MYR203) to Assess Safety and Efficacy of Myrcludex B in Combination with Peg-Interferon Alpha 2a in Patients with Chronic HBV/HDV Co-Infection. Hepatology 2018; 68 (Suppl): Abstract 16; https://doi.org/10.1016/S0618-8278(19)30141-0

16. Haag M, Hofmann U, Mürdter TE, et al. Quantitative bile acid profiling by liquid chromatography quadrupole time-offlight mass spectrometry: monitoring hepatitis B therapy by a novel Na-taurocholatecotransporting polypeptide inhibitor. Anal Bioanal Chem 2015; 407: 6815-25; https://doi.org/10.1007/s00216-015-8853-5

17. Blank A, Eidam A, Haag M, et al. The NTCP - inhibitor myrcludex B: effects on bile acid disposition and tenofovir pharmacokinetics. Clin Pharmacol Ther 2018; 103: 341-8; https:// doi.org/10.1002/cpt.744

18. Yuen MF, Locarnini S, Lim TH, et al. Short term RNA interference therapy in chronic hepatitis B using JNJ-3989 brings majority of patients to $\mathrm{HBsAg}<100 \mathrm{IUml}$ threshold [abstract PS-080]. J Hepatol 2019; 70: e51; https://doi.org/10.1016/S0618-8278(19)30092-1 
19. Agarwal K. Bi- weekly dosing of ARB-1467 LNP siRNA in HBeAg negative, virally suppressed patients with chronic HBV infection leads to deeper declines in $\mathrm{HBsAg}$ and potential association with IL28b [abstract]. Hepatology 2017; 66: LB17

20. Yuen MF, Agarwal K, Gane EJ, et al. Final Results of a Phase 1b 28-Day Study of ABI-H0731, a Novel Core Inhibitor, in Non-Cirrhotic Viremic Subjects with Chronic Hepatitis B. Hepatology 2018; 68 (S1): Abstract 73; https://doi.org/10.1016/S0168-8278(18)30439-2

21. Zoulim F, Yogaratnam J, Vandenbossche JJ, et al. Safety, pharmacokinetics and antiviral activity of a novel hepatitis b virus (HBV) capsid assembly modulator, JNJ-56136379, in Patients with Chronic Hepatitis B (CHB). Hepatology 2018; 68 (S1): Abstract 74; https://doi.org/10.1016/ S0168-8278(18)30423-9

22. Yuen MF, Kim DJ,Weilert F, et al.NVR 3-778, a First-in-Class HBV Core Inhibitor, Alone and in Combination with Peg-Interferon (PegIFN), in Treatment-Naive HBeAg-Positive Patients: Early Reductions in HBV DNA and HBeAg. J Hepatology 2016; 64 (Suppl): S210-S211; Abstract LBO6; https://doi.org/10.1016/S0168-8278(16)00175-6

23. Vaillant $A$, Bazinet $M$, Pantea $V$, et al. Updated follow-up analysis in the REP 401 protocol: Treatment $\mathrm{HBeAg}$ negative chronic hepatitis B infection with REP 2139 or REP 2165, tenofovir disoproxil fumarate and pegylated interferon alfa-2a.J Hepatol 2018; 68 (Suppl 1): S517; Abstract FRI-343; https://doi.org/10.1016/S0168-8278(18)31283-2

24. Vaillant A. Nucleic acid polymers: Broad spectrum antiviral activity, antiviral mechanisms and optimization for the treatment of hepatitis B and hepatitis D infection. Antiviral Res 2016; 133: 32-40; https://doi.org/10.1016/j.antiviral.2016.07.004

25. Bazinet M, Pântea V, Cebotarescu V, et al. Safety and efficacy of REP 2139 and pegylated interferon alfa-2a for treatment-naive patients with chronic hepatitis $\mathrm{B}$ virus and hepatitis $\mathrm{D}$ virus co-infection (REP 301 and REP 301-LTF): a non-randomised, open-label, phase 2 trial. Lancet Gastroenterol Hepatol 2017; 2: 877-89; https://doi.org/10.1016/S2468-1253(17)30288-1

26. Königer C, Wingert I, Marsmann M, et al. Involvement of the host DNA- repair enzyme TDP2 in formation of the covalently closed circular DNA persistence reservoir of hepatitis B viruses. Proc Natl Acad Sci USA 2014; 111: 4244-53; https://doi.org/10.1073/pnas.1409986111

27. Long Q, Yan R, Hu J, et al. The role of host DNA ligases in hepadnavirus covalently closed circular DNA formation. PLOS Pathog 2017; 13: e1006784; https://doi.org/10.1371/journal. ppat. 1006784

28. Seeger C, Sohn, JA. Complete spectrum of CRISPR/Cas9-induced mutations on HBV cccDNA. Mol Ther 2016; 7: 1258-66; https://doi.org/10.1038/mt.2016.94

29. Kennedy EM, Bassit LC, Mueller H, et al. Suppression of hepatitis B virus DNA accumulation in chronically infected cells using a bacterial CRISPR/Cas RNA-guided DNA endonuclease. Virology 2015; 476: 196-205; https://doi.org/10.1016/j.virol.2014.12.001

30. Sekiba K, Otsuka M, Ohno M, et al. Inhibition of HBV transcription from cccDNA with nitazoxanide by targeting the HBx-DDB1 interaction. Cell Mol Gastroenterol Hepatol 2019; 7: 297-312; https://doi.org/10.1016/j.jcmgh.2018.10.010

31. Sekiba K, Otsuka M, Ohno M, et al. Pevonedistat, a Neuronal Precursor Cell-Expressed Developmentally Down-Regulated Protein 8-Activating Enzyme Inhibitor, Is a Potent Inhibitor of Hepatitis B Virus. Hepatology 2019; 69: 1903-15; https://doi.org/10.1002/hep.30491

32. Rossignol JF, Bréchot C. A Pilot Clinical Trial of Nitazoxanide in the Treatment of Chronic Hepatitis B. Hepatol Commun 2019; 3: 744-7; https://doi.org/10.1002/hep4.1339

33. Nassal M. HBV cccDNA: viral persistence reservoir and key obstacle for a cure of chronic hepatitis B. Gut 2015; 64: 1972-84; https://doi.org/10.1136/gutjnl-2015-309809

34. Sato S, Li K, Kameyama T, et al. The RNA Sensor RIG-I Dually Functions as an Innate Sensor and Direct Antiviral Factor for Hepatitis B Virus. Immunity 2015; 42: 123-32; https://doi. org/10.1016/j.immuni.2014.12.016

35. Yuen MF, Chen CY, Liu CJ, et al. Ascending dose cohort study of inarigivir - a novel RIG I agonist in chronic HBV patients: final results of the ACHIEVE trial [abstract GS-12]. J Hepatol 2019; 70: e47-e48; https://doi.org/10.1016/S0618-8278(19)30084-2

36. Yuen MF, Elkhashab M, Chen CY, et al. Inarigivir demonstrates potent dose dependent antiviral activity in $\mathrm{HBV}$ treatment-naive patients: role of $\mathrm{HBeAg}$ status and baseline $\mathrm{HBsAg}$ in anti-viral response. Hepatology 2018; 68 (S1): Abstract 75

37. Menne S, Tumas DB, Liu KH, et al. Sustained efficacy and seroconversion with the Toll- like receptor 7 agonist GS-9620 in the Woodchuck model of chronic hepatitis B. J Hepatol 2015; 62: 1237-45; https://doi.org/10.1016/j.jhep.2014.12.026

38. Gane EJ, Lim YS, Gordon SC, et al. The oral Toll- like receptor-7 agonist GS-9620 in patients with chronic hepatitis B virus infection.J Hepatol 2015; 63: 320-8; https://doi.org/10.1016/j. jhep.2015.02.037 
39. Boni C, Vecchi A, Rossi M, et al.TLR7 agonist increases responses of hepatitis B virus- specific $\mathrm{T}$ cells and natural killer cells in patients with chronic hepatitis $\mathrm{B}$ treated with nucleos $(\mathrm{t})$ ide analogues. Gastroenterology 2018; 154: 1764-77; https://doi.org/10.1053/j.gastro.2018.01.030

40. Gane E, Kim HJ, Visvanathan K, et al. Safety, pharmacokinetics and pharmacodynamics of oral TLR8 agonist GS-9688 in patients with chronic hepatitis B: a randomized, placebo-controlled, double-blind Phase 1b study. Hepatology 2018; 68 (S1): Poster \#0401

41. Lok AS, Pan CQ Han SH, et al. Randomized phase II study of GS-4774 as a therapeutic vaccine in virally suppressed patients with chronic hepatitis B.J Hepatology 2016; 65: 509-16; https://doi.org/10.1016/j.jhep.2016.05.016

42. Gane E, Gaggar A, Nguyen AH, et al. A phase1 study evaluating anti-PD-1 treatment with or without GS-4774 in HBeAg negative chronic hepatitis B patients. J Hepatology 2017; 66 (Supplement): S26-S27; https://doi.org/10.1016/S0168-8278(17)30315-X

43. Gane E, Verdon DJ, Brooks AE, et al. Anti-PD-1 blockade with nivolumab with and without therapeutic vaccination for virally suppressed chronic hepatitis B: A pilot study. J Hepatology 2019; 71: 900-7; https://doi.org/10.1016/j.jhep.2019.06.028

44. Cerino A, Mantovani S, Mele D, et al. Human Monoclonal Antibodies as Adjuvant Treatment of Chronic Hepatitis B Virus Infection. Front Immunol 2019; 10:2290; https://doi.org/10.3389/ fimmu.2019.02290

45. Zhang T-Y, Yuan Q, Zhao J-H, et al.Prolonged suppression of HBV in mice by a novel antibody that targets a unique epitope on hepatitis B surface antigen. Gut 2016; 65: 658-71; https://doi. org/10.1136/gutjnl-2014-308964

46. Li D, He W, Liu X, et al. A potent human neutralizing antibody Fc-dependently reduces established HBV infections. Elife 2017; 6: e26738; https://doi.org/10.7554/eLife.26738

47. Bertoletti A, Tan AT, Koh S. T-cell therapy for chronic viral hepatitis. Cytotherapy 2017; 19: 1317-24; https://doi.org/10.1016/j.jcyt.2017.07.011

48. Boni C, Barili V, Acerbi G, et al. HBV Immune-Therapy: From Molecular Mechanisms to Clinical Applications. Int J Mol Sci 2019; 20: pii: E2754; https://doi.org/10.3390/ijms20112754 